\title{
Necesidades de cuidado dentro del hospital del cuidador de personas con cancer
}

\author{
Care Needs of Caregivers of Patients with Cancer during Inpatient Stay \\ Necessidades de cuidado no âmbito hospitalar do cuidador de pessoas \\ com câncer
}

\begin{abstract}
Como citar este artículo
Lins, Ana Luiza Rodrigues; Mendonça, Erica Toledo de; Moreira, Tiago Ricardo; Matos, Renata Almeida de; Andrade, João Vitor; Martins, Thalyta Cássia de Freitas; Carmo, Monica Maria Lopes do. Necesidades de cuidado dentro del hospital del cuidador de personas con cancer. Revista Cuidarte. 2021;12(2):e1231.

http://dx.doi.org/10.15649/cuidarte.1231
\end{abstract}

Revista Cuidarte

Rev Cuid. May - Ago 2021; 12(2): e1231

doi" http://dx.doi.org/10.15649/cuidarte.1231

E-ISSN: 2346-3414

(1) Ana Luiza Rodrigues Lins ${ }^{1}$

(1) Erica Toledo de Mendonça ${ }^{2}$

(1) Tiago Ricardo Moreira ${ }^{3}$

(1) Renata Almeida de Matos ${ }^{4}$

(1) João Vitor Andrade ${ }^{5}$

(1) Thalyta Cássia de Freitas Martins ${ }^{6}$

(1) Monica Maria Lopes do Carmo

1 Instituto Nacional do Câncer, Rio de Janeiro, Brasil.

E-mail: luizalyralins@gmail.com

2 Universidade Federal de Viçosa, Viçosa, Minas Gerais, Brasil. E-mail: ericaenfufv@gmail.com Autor de Correspondencia

3 Universidade Federal de Viçosa, Viçosa, Minas Gerais, Brasil. E-mail: tiagoricardomoreira@gmail.com

4 Fundação Cristiano Varella, Muriaé, Minas Gerais, Brasil.

E-mail: r.matos@fcv.org.br

5 Universidade de São Paulo, São Paulo, Brasil.

E-mail: jvma100@gmail.com

6 Universidade Federal de Viçosa, Viçosa, Minas Gerais, Brasil. E-mail: enfermeirathalyta@gmail.com

7 Universidade de São Paulo,São Paulo, Brasil. E-mail:

monicacarmolopesmaria@gmail.com

\section{Resumen}

Introducción: La existencia de un paciente con cáncer en el seno de la familia implica desafíos y lleva a una necesidad de reorganización de la dinámica familiar para la prestación de cuidados, lo que puede provocar la negligencia de los auto-cuidados del cuidador, además de las necesidades de segundarización dentro del hospital, durante la hospitalización del ente enfermo. Objetivo: comprender qué cuidados los familiares cuidadores de personas con cáncer gustarían recibir en el hospital. Materiales y Métodos: estudio cualitativo, realizado en un hospital de cáncer de Brasil, con 14 familiares, en 2019. Las entrevistas fueron cerradas en el momento en que existía la saturación de datos y analizadas por la técnica de Análisis de Contenido. La investigación cumple con los aspectos éticos. Resultados: la primera categoría reveló el acogimiento, las competencias de actitud del equipo, la asistencia psicológica y la casa de apoyo como medidas importantes que la institución ofrece a los cuidadores. La segunda categoría mostró qué cuidados los familiares gustarían recibir, y los testimonios revelaron la transferencia del cuidado de sí mismo a los demás, y aún las demandas de cuidado físico, espiritual y psicológico. Discusión: es evidente que existe un acercamiento entre lo que el cuidador espera de la institución estudiada como cuidado de sí mismo, con la asistencia ofrecida. Conclusiones: el estudio señala la necesidad de planificar estrategias de apoyo a los familiares cuidadores en el entorno hospitalario, además de grupos de apoyo interdisciplinario y apoyo psicológico continuo.

Palabras clave: Cuidadores; Neoplasias; Enfermería; Relaciones Familiares.

Recibido: mayo 1 de 2020

Aceptado: febrero 2 de 2021

Publicado: abril 30 de 2021 $\square *$ Correspondencia

Erica Toledo de Mendonça

E-mail: ericaenfufv@gmail.com 


\title{
Care Needs of Caregivers of Patients with Cancer during Inpatient Stay
}

\begin{abstract}
Introduction: Having a patient with cancer in the family entails challenges leading to a need to reorganize family dynamics to provide care, which may result in neglecting caregiver's selfcare and their needs at the hospital during inpatient stay. Objective: To understand how family caregivers would like to be cared for during hospital stay. Materials and Methods: A qualitative research was conducted with 14 family members in a Brazilian oncology hospital in 2019. Interviews were ended upon data saturation and later analyzed using the Content analysis method. Ethical aspects were considered in this research. Results: The first category showed that welcoming, attitudinal competencies of the healthcare team, psychological support and home support are considered important measures that healthcare centers can provide caregivers. The second category identified how family members would like to be cared for through interviews, revealing care transference from oneself to the care of another, as well as physical, spiritual and psychological care demands. Discussion: Our understanding is that care expectations of caregivers in the above healthcare center are close to the actual assistance offered to them. Conclusions: A need for planning support strategies for family caregivers during inpatient stay has been pointed out in this study, in addition to the importance of interdisciplinary support groups and continuous psychological support that cover family members as part of these care programs.
\end{abstract}

Key words: Caregivers; Neoplasms; Nursing; Family Relations.

\section{Necessidades de cuidado no âmbito hospitalar do cuidador de pessoas com câncer}

\section{Resumo}

Introdução: A existência de um doente com câncer no seio familiar implica em desafios e conduz a uma necessidade de reorganização da dinâmica familiar para prestação de cuidados, o que pode ocasionar, na negligência do autocuidado do cuidador, além da secundarização das necessidades deste no âmbito hospitalar, durante internação do ente adoecido. O objetivo é compreender como os familiares cuidadores de pessoas com câncer gostariam de ser cuidados no cenário hospitalar. Materiais e Métodos: pesquisa qualitativa, realizada em um hospital oncológico brasileiro, com 14 familiares, em 2019. As entrevistas foram encerradas no momento em que houve saturação de dados, e analisadas pela técnica de Análise de Conteúdo. A pesquisa respeitou os aspectos éticos. Resultados: a primeira categoria revelou que o acolhimento, competências atitudinais da equipe, acompanhamento psicológico e a casa de apoio como medidas importantes que a instituição oferece aos cuidadores. A segunda categoria apresentou como os familiares gostariam de ser cuidados, e os depoimentos revelaram a transferência do cuidado de si para o outro, e ainda demandas de cuidado no âmbito físico, espiritual e psicológico. Discussão: depreende-se que existe uma aproximação entre o que o cuidador espera da instituição estudada como cuidado para si, com a assistência que lhe é oferecida. Conclusões: $O$ estudo apontou para a necessidade de planejamento de estratégias de apoio aos familiares cuidadores no âmbito hospitalar, além de grupos de apoio interdisciplinares e suporte psicológico contínuo, incluindo os familiares no plano de cuidados.

Palavras chave: Cuidadores; Neoplasias; Enfermagem; Relações Familiares. 


\section{Introducción}

El cáncer se ha configurado como un importante problema de salud pública, y se espera la presencia de 20 millones de nuevos casos en el mundo, con el $80 \%$ de ellos ocurriendo en los países en desarrollo'. Se estima para el trienio 2020-2022 en Brasil la ocurrencia de 625 mil nuevos casos, con las muertes causadas por el cáncer ocupando la segunda causa de muerte².

Sin embargo, a pesar de los avances en los métodos de la detección precoz del cáncer, aún existe un alto porcentaje de casos cuyos diagnósticos son tardíos, repercutiendo en la supervivencia del paciente, configurando un peor pronóstico de la enfermedad, así como interfiriendo directamente en la dinámica familiar, ya que implica una mayor necesidad de atención por parte de la familia ${ }^{3}$.

Segundo Boff ${ }^{4}$, el cuidado si muestra cómo una actitud de relación amorosa, gentil, amable, armoniosa y protectora para la realidad personal, social y ambiental. Por lo tanto, el cuidado revela que el otro tiene importancia porque promueve la participación con su vida y su destino.

A partir de estas consideraciones, es importante destacar que la enfermedad de un miembro familiar hace que se movilicen para buscar estrategias para producir y administrar los cuidados de rutina, a fin de satisfacer las necesidades de aquello, que modifica la estructura familiar, generando consecuencias. Junto a este cambio en la dinámica familiar, hay el estigma asociado con el diagnóstico de cáncer, repercutiendo en sentimientos de temor, incertidumbre de la curación y el sufrimiento ante la situación vivida ${ }^{5}$.

De esta manera, muchas veces la experiencia de cuidar a un familiar enfermo de cáncer representa una gran carga física, emocional y financiera para el núcleo familiar, visto que requiere disponibilidad, tiempo y dedicación, y puede resultar en la enfermedad del cuidador, conduciendo a la depresión, la ansiedad, la fatiga mental y física ${ }^{6}$. Estos efectos fueron observados en un estudio que mostró un desequilibrio en el mantenimiento de las necesidades humanas básicas
La experiencia de cuidar a un familiar enfermo de cáncer representa una gran carga física, emocional y financiera para el núcleo familiar, visto que requiere disponibilidad, tiempo y dedicación, y puede resultar en la enfermedad del cuidador, conduciendo a la depresión, la ansiedad, la fatiga mental y física ${ }^{6}$ de los familiares entrevistados, con cambios en las dimensiones físicas, espirituales y sociales, y sentimientos negativos como el miedo, el shock, la tristeza y el sufrimiento ${ }^{5}$.

En este contexto, surge la necesidad de apoyo profesional a los miembros de la familia, a fin de aliviar el desgaste sufrido por estos individuos durante el proceso de la enfermedad de su familiar $^{3}$. En este contexto, se destaca el papel estratégico de los profesionales de enfermería junto a los familiares cuidadores de personas con cáncer, ya que están en contacto directo con el núcleo familiar enfermo, convirtiéndose en actores esenciales para la viabilidad de las medidas que mitiguen la sobrecarga de éstos.

En este sentido, las siguientes preguntas guiaron la investigación: ¿Cuáles estrategias de cuidado son ofrecidas a los familiares cuidadores en un hospital de referencia en Oncología? ¿Qué cuidado los familiares cuidadores de pacientes con cáncer gustarían recibir? ¿Qué estrategias pueden ser realizadas por los profesionales de Enfermería para suavizar y reducir la sobrecarga de estos cuidadores?

Se verificó una escasez en la literatura de estudios abordando estrategias de actuación con los familiares de personas con cáncer, con el objetivo de reducir la carga de estos cuidadores. Así, el 
objetivo de esta investigación es entender qué cuidados los familiares cuidadores de personas con cáncer gustarían recibir en el contexto hospitalario.

\section{Materiales y Métodos}

Investigación cualitativa, que trabaja con el universo de creencias, valores y actitudes de los sujetos incluidos en una determinada realidad social, que incluye a los fenómenos humanos. Estudios de esta naturaleza toman en cuenta las cuestiones de la existencia humana, considerando la subjetividad de los problemas, profundizando en los significados que las personas atribuyen a sus acciones y relaciones humanas ${ }^{7}$.

La investigación ocurrió en la Clínica Médica de un hospital brasileño de cáncer. Los participantes de la investigación fueron los principales familiares cuidadores de personas con cáncer que estuviesen como acompañantes, con 18 años o más, cuyo miembro familiar tuviese recibido el diagnóstico de cáncer hace más de 6 meses, justificado como un período en el que ya se han experimentado momentos importantes de diagnóstico y tratamiento por parte del núcleo familiar.

El equipo de investigación estuvo compuesto por dos investigadores, uno estudiante de enfermería y otro docente, con experiencia en investigación cualitativa. Antes de la recolección de datos, el maestro capacitó al estudiante en la técnica de recolección de datos de la entrevista individual y se registró en un diario de campo. Además, el campo de investigación se definió antes del inicio de las entrevistas, y se estableció contacto con él para establecer antes de iniciar la investigación.

La recogida de datos se produjo a través de entrevistas en sala reservada y se guio por un guía conteniendo datos sociodemográficos y relativos a la persona con cáncer, y preguntas abiertas sobre el tema. Se utilizó un registrador para registrar la información recolectada, con una duración promedio de treinta a cuarenta minutos por participante. Por tanto, buscamos anotar las impresiones y sentimientos del investigador en un diario de campo. Los participantes fueron identificados por la letra C (Cuidador), para preservar el anonimato.

La recogida de datos se produjo en 2019 y fue cerrada en el momento en que existía la saturación de datos. El criterio de cierre de muestreo por saturación teórica se produce en el momento en que se detiene la recopilación de datos cuando se observa que los nuevos elementos para subvencionar la teorización no son más deprendidos del campo de observación ${ }^{8}$. Finalmente, para la transcripción y análisis de datos, no usó software para la gestión, transcripción de datos.

El análisis de las entrevistas se realizó mediante la técnica de análisis de contenido de Lawrence Bardin, que propone una secuencia de análisis basada en las siguientes etapas: Pre-análisis, exploración del material y tratamiento de los resultados, inferencia e interpretación. Inicialmente, se hace una lectura flotante y exhaustiva de las cuestiones de las entrevistas. A continuación, se procede a la selección temática, que consiste en identificar los núcleos de significado, o elementos con semántica similar, para su posterior clasificación, tratamiento de los resultados, inferencia e interpretación9.

Este estudio es parte de un estudio mayor titulado "Evaluación de la sobrecarga de los familiares cuidadores de pacientes con cáncer", desarrollado de acuerdo con los aspectos éticos de la Resolución 466/2012 del Consejo Nacional de Salud de Brasil ${ }^{10}$. Fue aprobado por el Comité de Ética en Investigación con Seres Humanos de la universidad pública a la que esta investigación es vinculada. 


\section{Resultados}

\section{Caracterización de los participantes}

De los 14 participantes en el estudio, la prevalencia fue del sexo femenino, haciendo un total de 10 mujeres $(71,4 \%)$ y cuatro hombres $(28,5 \%)$. En relación a la edad, hubo una variación de 23 a 56 años, con un promedio de 45,7 años. En cuanto al grado de escolaridad, el mismo fue evaluado en años de estudio y osciló entre 6 años y 21 años, con un promedio de 11,7 años. El grado de parentesco presentó un rango entre hijastros $(7,1 \%)$, hermanos y sobrinos $(14,2 \%)$, niños $(35,7 \%)$ y mujeres (28,5\%). Del total de participantes, el 92,8\% informó participar directamente en el cuidado del familiar enfermo, y el período de tiempo como cuidador osciló entre un mínimo de seis meses y un máximo de dieciséis años.

\section{Cuidar a los que cuidan: estrategias de apoyo para familiares cuidadores}

Esta categoría muestra las principales estrategias de apoyo proporcionadas por el equipo de salud de la institución estudiada, de acuerdo con los familiares cuidadores de personas con cáncer. El análisis de las declaraciones demostró que los familiares citaron como estrategias de apoyo el acogimiento, refiriéndose a las competencias de las actitudes del equipo de salud, así como el apoyo psicológico, la ayuda de equipo en el cuidado directo al familiar enfermo y la casa de apoyo de la institución.

\author{
los familiares citaron como \\ estrategias de apoyo el \\ acogimiento, refiriéndose a las \\ competencias de las actitudes del \\ equipo de salud, así como el apoyo \\ psicológico, la ayuda de equipo \\ en el cuidado directo al familiar \\ enfermo y la casa de apoyo de la \\ institución.
}

Las declaraciones siguientes se refieren al acogimiento practicado por el equipo, e indican las aptitudes de actitudes como la simpatía, el afecto, la atención, la responsabilidad, como forma importante de abordaje junto a los familiares cuidadores: "[...] son agradables, sonrientes" (C1). "A veces mi madre está a caer acá, [...] ellos suben, pasan la mano y resuelven." (C5); "[...] Son todos cariñosos" (C5); "[...] entonces, creo que la atención aquí es muy elogiable" (C13); "[...] si necesario, los llamamos y ellos vienen de inmediato, y le ayudan [...]"; "[...] son profesionales muy responsables [...]" (C14); "[...] ellos abrazan, ¿sabes? Si verme llorar, o allí dentro del cuarto con algo, todo el mundo quiere averiguar qué está pasando. [...] (C9).

También se observó en las declaraciones de los participantes que el apoyo psicológico fue nombrado como otra estrategia importante de apoyo a los familiares, como se observa en los fragmentos que siguen: "[...] un atendimiento psicológico [...] algunos ya hablaron a mí. Esto ayuda mucho" (C3); "[...] en la parte de psicología ella cuida muy bien [...] charlar con usted, le ayudan a comprender, nos ayudan a comprender todo lo que está pasando. ¡Es óptimo!" (C9).

Otra cuestión que apareció en las declaraciones de los entrevistados fue que muchas veces el apoyo ofrecido por el equipo se direccionaba a las directrices relativas al cuidado y/o estado de salud del paciente, y no necesariamente con un enfoque en las necesidades del familiar cuidador. Esos resultados son ratificados por las siguientes declaraciones: "[...] tiene también apoyo de la enfermera jefe, ella habla y explica las cosas, da las directrices del baño, del remedio"(C10); "[...] si sé de todo lo que está ocurriendo, si estoy dentro de todo lo que está sucediendo con mi esposo" (C9).

Y finalmente, el apoyo fue citado por muchos cuidadores como estrategia de cuidado ofrecida por la institución a los familiares. Siguen los testimonios que indican esta cuestión: "[...] ellos 
tienen un equipo de servicio personalizado, especialmente si la persona tiene cáncer en fase terminal, ya encamina al tratamiento psicológico. ;También tiene un equipo de acompañamiento espiritual que actúa en la espiritualidad, lo que ayuda mucho! Y tiene un equipo de alegría que hace un trabajo maravilloso" (C14); "[...] si alguien precisar permanecer aquí en el hospital cuenta con la casa de apoyo" (C13).

\section{Qué cuidados los familiares cuidadores gustarían recibir: expectativas sobre el cuidado de si}

Esta categoría muestra las necesidades de cuidado de los familiares cuidadores de personas con cáncer. Las declaraciones revelaron la transferencia del cuidado de sí a los otros, y aún las demandas de cuidado físico, espiritual y psicológico.

Cuando se preguntó acerca de cómo les gustaría ser cuidados, se observó que muchos de los participantes no se veían como sujetos que demandan cuidados, siendo el foco de atención en otro, es decir, en los familiares enfermos. Estas preguntas pueden ser constatadas en las declaraciones que siguen: "para mí, no pido mucho no. Tengo salud para cuidar de su salud, que es la importante[...]" (C3); "[...] Hacer lo que hacen a mi madre, hacen por mí[...]encontrar un tratamiento, igual es a mi madre, y ella es tan bien tratada y ¿usted aún piensa en mí?[...]quizá pedir demasiado"(C5); "[...] ;Creo que es más difícil, porque ya están cuidando del paciente, y tienen que mirar pro lado que necesita más![....]" (C12).

También se constató que estos cuidadores, muchas veces, si cancelaron en favor del otro, una vez que la participación en el cuidado de sus familiares fue colocado como una prioridad. El tiempo antes dedicado a la realización de diversas actividades, incluyendo el cuidado de su salud, ahora era totalmente dedicado al cuidado del familiar enfermo. Además, estos cuidadores estaban en un conflicto entre el cuidado de si y del otro. Los fragmentos a continuación ilustran estas cuestiones: "[...] tiene un día que pienso: "¿Dónde estoy? ¿Quién soy yo? ¿Lo que estoy haciendo aquí? Por lo tanto, como ustedes saben, es que parece que el cuerpo sale de nosotros [...] (C5)"; "[...] me siento así bien porque estoy ayudándola, y mal porque estoy mí dejando de lado" (C8); "[...] Porque estamos presos y no presos" (C6).

Además, uno de los entrevistados mencionó que el acto de hablar con otros cuidadores era una medida de apoyo; la declaración que sigue ilustra este hallazgo: "[...] a veces estás en el pasillo, una conversación, un asunto, relatar el sufrimiento del paciente que acompañan, entonces usted relata su. Lo que también le ayuda un poco. Un da soporte pro otro" (C3).

Por otro lado, las declaraciones que siguen indican las necesidades de cuidado relatadas por los familiares cuidadores en los ámbitos físico, espiritual y psicológico: "[...] que las personas recen por mí para Dios me dar fuerza [...]" (C12); "[...] como, por ejemplo, ellos

Las declaraciones que siguen indican las necesidades de cuidado relatadas por los familiares cuidadores en los ámbitos físico, espiritual y psicológico. permiten la iglesia entrar aquí. Católica, evangélica, yo soy católica. Pero es una manera de cuidar de las personas, una manera de tratar de las personas." (C6). "[...] y yo creo que el cuerpo necesita un ejercicio, necesita trabajar la cabeza, e independiente de la enfermedad. Necesitamos, creo que ayudaría también" (C5); "[...] también podría tener un espacio para los cuidadores consultaren el médico si sienten algo. Porque nosotros, como cuidadores, enfermos, no funciona" (C1). 
Más allá de lo mencionado, cabe señalar que muchos de los participantes informaron que la relación interpersonal impregnada por el acogimiento, la aproximación con los otros, escucha, atención, conversar, ser atento y cauteloso, son importantes estrategias de cuidado al cuidador. Estas conclusiones se expresan en las declaraciones que siguen: "[...] $a$ veces conversar, abrazar [...]"(C5); "[...] abrazaba, preguntándome cómo estaba, entonces, esta forma de tratamiento ayuda a las personas tan bien" (C6); "[...]hay personas que están más necesitadas y necesitan atención [...]" (C13); "Ha de ser más humanos, más próximo del paciente, y es una área que la aproximación del otro hace la diferencia. [...] La competencia técnica, la persona estudia y da cuenta de ello, pero la parte humana, tiene que ser trabajada día a día, es en el contacto [...]" (C14).

\section{Discusión}

El análisis de los datos reveló que el mayor porcentaje de familiares cuidadores de personas con cáncer era femenino, un hallazgo que está en consonancia con la literatura, que retrata a la mujer como figura referencial en la atención, así como las funciones que ejerce sobre una base diaria en casa ${ }^{11}$. En relación con el lazo los familiares, la mayoría eran niños y cónyuge, confirmando los hallazgos de la literatura, que muestran que el familiar cuidador son personas de la misma familia y que tienen una mayor relación con el familiar enfermo ${ }^{12}$.

El análisis de los resultados de la primera categoría indica que el acogimiento celebrado por los profesionales de salud, refiriéndose a las competencias de actitud del equipo de competencias, fue considerado como una importante estrategia para apoyar a los familiares cuidadores de personas con cáncer. De acuerdo con la Política Nacional de Humanización (PNH) de Brasil, se

El acogimiento celebrado por los profesionales de salud, refiriéndose a las competencias de actitud del equipo de competencias, fue considerado como una importante estrategia para apoyar a los familiares cuidadores de personas con cáncer. entiende el acogimiento como una práctica constructiva de las relaciones de cuidado, apuntando a la creación de lazos, promoviendo la escucha terapéutica, permitiendo que la persona pueda exponer sus demandas y, por lo tanto, facilitando la resolución de problemas ${ }^{13}$.

En este sentido, los cuidadores destacaron las actitudes del equipo de salud como importantes medidas de apoyo, entre ellas el acogimiento y el hecho de estar atentos a sus necesidades, haciendo hincapié los resultados de la literatura. La investigación muestra que es de suma importancia la aplicación del acogimiento y la provisión de información al familiar cuidador, principalmente en el medio hospitalario, apuntando a una asistencia integral para el núcleo familiar enfermo ${ }^{14}$.

Esto es particularmente importante en el contexto de cáncer que muestra, en la mayoría de los casos, los tratamientos complejos que requieren muchas hospitalizaciones y acciones dirigidas al núcleo familiar enfermo que minimicen el impacto de la enfermedad. Por lo tanto, el impacto psicológico de estas cuestiones en el cuidador requiere acciones como la escucha activa, la prestación de ayuda y la provisión de orientaciones, entre otros, con el fin de transformar en un ambiente acogedor y humanizado, ayudando en el proceso de enfrentamiento de la enfermedad ${ }^{15}$. 
Además, el acogimiento citado por los participantes es una forma de alentar el establecimiento de vínculos con esos parientes, desarrollando una relación de confianza, lo que facilita la comunicación entre las partes. En este sentido, una comunicación clara es una habilidad esencial en cualquier tipo de relación, ya que mantiene al paciente y a sus cuidadores informados sobre el tratamiento y las necesidades de cuidados con el familiar enfermo, además de reducir los temores y las dudas que surgen en el curso del tratamiento ${ }^{16}$.

Aún en esta categoría, el psicológico fue nombrado como estrategia de apoyo importante, proporcionando al familiar cuidador una manera más eficaz para lidiar con las emociones surgidas del proceso de cuidar a una persona con cáncer. Un estudio brasileño ha identificado que los familiares de las personas con cáncer tienden a experimentar los mismos sentimientos que las personas diagnosticadas con cáncer, tales como el miedo, la angustia y la tristeza, los cuales pueden llevar a una angustia psicológica ${ }^{17}$. Por lo tanto, el acto de conversar y recibir la atención de un profesional capacitado para lidiar con estas exigencias se configura como una medida de atención con el cuidador ${ }^{18}$. El cuidado psicológico no debe ser una medida puntual, sino continua, con el fin de minimizar el shock emocional derivado del cambio en la dinámica familiar y la sobrecarga de tareas del familiar cuidador ${ }^{19}$.

Otra estrategia importante de cuidado al cuidador mencionada por los entrevistados fue la Casa de Apoyo de la institución, escenario de esta investigación. En esta ubicación, los pacientes y sus cuidadores puedan permanecer alojados en conformidad con el tratamiento de rutina, donde reciben alimentos, transporte institucional, servicio de lavandería, zona de recreo, así como grupos de costura, artesanía y otros, además de ser asistidos por un equipo multiprofesional sobre una base semanal.

Estudio retrató la casa de apoyo como una importante forma de acogimiento y cuidado de los familiares cuidadores de personas con cáncer, describiendo la asistencia humanizada, empática y plena de los profesionales de la salud con estos individuos, corroborando los resultados del presente estudio ${ }^{20}$.

Las conclusiones de la segunda categoría, que identificó qué cuidados los cuidadores familiares gustarían recibir, encontraron que muchos de los participantes no se veían como sujetos que exigen cuidado, siendo el foco de atención sólo en el familiar enfermo. Sobre esta cuestión, el autor que sigue corrobora los hallazgos del presente estudio: se consideró implícita en sus lenguas la renuncia de la propia rutina, sus hábitos y costumbres, privándose de sus necesidades existenciales en favor del restablecimiento de la salud de sus familiar enfermo, porque entendieron que cumplir con las exigencias impuestas por la enfermedad se vuelve más urgente en relación a otras áreas de su vida ${ }^{19}$.

Así, constatando los resultados de arriba, los estudios indican que los familiares cuidadores de personas afectadas por cualquier neoplasia viven momentos estresantes, impregnados por angustias y aflicciones, y por estar en contacto directo con el familiar enfermo, tienden a experimentar los mismos dolores y preocupaciones del otro. Estos percances culminan en el descuido del cuidador a su propia salud, porque priva su estimulación y tiempo para llevar a cabo acciones en favor de su auto-cuidado ${ }^{20-22}$.

Por otro lado, se plantearon las expectativas sobre el cuidado de sí por los participantes el contexto físico, espiritual y psicológico, y estos resultados están en consonancia con la 
literatura. Los estudios demuestran que la práctica de actividades de ocio, tales como la actividad física, es una forma de minimizar la sobrecarga de funciones asignadas a los cuidadores, pues está relacionada con la reducción de estrés y también al aumento de la autoestima. Esta acción favorece la reducción de las emociones negativas vivenciadas por los familiares cuidadores durante el tratamiento y, por consiguiente, se traduce en una mejor calidad de vida ${ }^{10,12}$.

Además, la realización de actividades físicas como el estiramiento y la relajación puede ayudar al individuo a volver la mirada para sí, permitiendo el contacto con el propio cuerpo y despertando a la importancia de cuidar de sí mismo. De esta manera, estas medidas ayudan a reducir el sufrimiento del cuidador, así como la promoción de la salud ${ }^{23}$.

También en este contexto, algunos encuestados señalaron como demanda de cuidado el apoyo médico, teniendo en cuenta que, a fin de ofrecer un cuidado de calidad a sus parientes enfermos, es imperativo que los cuidadores tengan condiciones de salid adecuadas, un hallazgo que corrobora los datos de la literatura ${ }^{24}$.

Por otra parte, la necesidad de apoyo espiritual fue señalada como una demanda de cuidado. Estudio muestra que la religiosidad es una medida de apoyo, debido a que promueve el contacto con las personas y situaciones que pueden ofrecer apoyo espiritual a los temores y ansiedades que a menudo viven ${ }^{22}$.

Otro estudio determinó la espiritualidad como una importante herramienta de enfrentamiento de las adversidades del cuidado con el familiar enfermo ${ }^{13}$. Este estudio demostró también que cuidar de los otros es una manera de replantear el contexto vivido,

La espiritualidad como una importante herramienta de enfrentamiento de las adversidades del cuidado con el familiar enfermo ${ }^{13}$ que también contribuye a la (re)orientación de las formas de cuidado. Ratificando los estudios mencionados y confirmando los resultados del presente estudio, Machado ${ }^{22}$ enumera en su tesis la espiritualidad como un factor protector de la salud, pues el hecho de que las personas poseen una creencia/religión desalienta las actitudes perjudiciales para la calidad de vida, sino que también favorece la reducción de los síntomas como la ansiedad, la angustia y el miedo.

En este sentido, es importante que el equipo de salud sea sensible a las cuestiones que impregnan la dimensión espiritual y religiosa de los pacientes y sus familiares cuidadores, con el fin de abordarlas en su práctica de atención. Por lo tanto, el profesional de enfermería debería ampliar su visión sobre lo que es invisible al ojo humano, es decir, acerca de la subjetividad/ necesidades presentadas por el familiar cuidador, que a menudo se encuentran en un plano secundario, sin limitar su asistencia a lo que ven.

Con relación al apoyo psicológico, esto fue listado por la gran mayoría de los participantes como una medida esencial en la gestión del impacto del cuidado y significativa estrategia de apoyo, como se señaló en la primera categoría. Cabe señalar que el apoyo psicológico fue insertado por el Ministerio de Salud brasileño como una asistencia obligatoria durante el tratamiento oncológico, debido al hecho de que el cáncer puede generar desequilibrio emocional en el 
núcleo familiar enfermo ${ }^{3,25}$. En el ínterin, el equipo de salud debe proporcionar la supervisión basada en ofrecer apoyo, atención, comprensión de los sentimientos surgidos y las directrices relativas al tratamiento, siendo estas las medidas pertinentes para minimizar el impacto emocional.

Así, el acto de hablar con otros cuidadores fue citado como una medida de enfrentamiento y alivio. Es importante hacer hincapié en la importancia de los grupos de apoyo como acción significativa para

Es importante hacer hincapié en la importancia de los grupos de apoyo como acción significativa para apoyar el familiar cuidador. apoyar el familiar cuidador. El grupo operativo es una técnica de grupo de intervención, donde las personas con un objetivo común se encuentran y viven momentos, donde comparten experiencias con personas que se encuentran en situaciones similares y replantean sus experiencias, pensamientos y emociones ${ }^{21}$.

Más allá de lo mencionado, cabe señalar que muchos de los participantes sostuvieron que la relación interpersonal como el acogimiento, la aproximación con los otros, escuchar, hablar, ser atento y cauteloso, son importantes estrategias de cuidado para el cuidador. Este hecho confirma los hallazgos en la literatura que traen como de vital importancia el desempeño de profesionales entrenados para identificar las demandas de cuidado biopsicosocial presentadas por estos cuidadores, ya que la calidad de vida del cuidador influirá directamente en la asistencia prestada a su familiar enfermo ${ }^{21}$.

\section{Conclusiones}

A partir de los hallazgos de la investigación, se infirió que existe una aproximación entre lo que el cuidador espera de los profesionales del equipo de salud de la institución como cuidado a sí mismos, con la asistencia que se ofrece.

El presente estudio ha identificado que el acogimiento, el apoyo psicológico y la Casa de Apoyo de la institución, escenario de la investigación, fueron identificados como medio de apoyo biopsicoespiritual a los cuidadores. Con respecto a los cuidados que gustarían recibir, muchos de los cuidadores no se veían como sujetos que exigían cuidados, siendo el enfoque de la asistencia en el familiar enfermo.

Frente a este escenario, los resultados de este estudio apuntan a la necesidad de desarrollar estrategias de apoyo a los familiares cuidadores de personas con cáncer de manera holística, acogedora y humanizada, con énfasis a las acciones direccionadas a cuidados en salud y morbilidad que los familiares presentan, grupos de apoyo en el escenario hospitalario, además de apoyo psicológico continuo, incluyendo a la familia en el plan de cuidados.

Vale la pena recordar que el cuidado va más allá de las acciones técnicas; él está presente en la mirada atenta, en el afecto, en el acto de acoger y escuchar a los demás, siendo estos expresiones del cuidar de las esencias de la práctica de Enfermería y de gran importancia para la recuperación y el mantenimiento del bienestar del núcleo familiar enfermo.

Otros estudios deben ser realizados en esta área en relación a los cuidados que las personas con cáncer gustarían recibir, con el fin de orientar la planificación de acciones englobando todos los individuos involucrados en el proceso de enfermedad por cáncer en el plan de cuidados. 
Declaración de Conflicto de Intereses: o los autores declaran que no hay conflictos de interés.

Financiacion: Fundação de Amparo à Pesquisa do Estado de Minas Gerais (FAPEMIG), Minas Gerais, Brasil.

\section{Referencias}

1. Globocan. Estimaded Cancer Incidence, Mortality and Prevalence Worldwide in International Agency for Research on Cancer. World Health Association; 2012. Disponible: http://globocan.iarc.fr/Default.aspx

2. INCA (Instituto Nacional de Câncer). Brasil. Estimativa 2020/2022: incidência de câncer no Brasil. Instituto Nacional do Câncer: Rio de Janeiro; 2019. Disponible: https://www.inca.gov. br/sites/ufu.sti.inca.local/files//media/document//estimativa-2020-incidencia-de-cancerno-brasil.pdf

3. Petean E, De Araujo LFS, Bellato R. Dimensão espaço-tempo e os atos-atitudes de cuidado na experiência familiar. Rev. Cuidado Fundam. Care. 2016;8(3):4738-48.

http://dx.doi.org/10.9789/2175-5361.2016.v8i3.4738-4748

4. Boff L. O cuidado necessário: Na vida, na saúde, na educação, na ecologia, na ética e na espiritualidade. Rio de Janeiro: Editora Vozes; 2012.

5. Delalibera M, Presa J, Barbosa A, Leal I. Sobrecarga no cuidar e suas repercussões nos cuidadores de pacientes em fim de vida: revisão sistemática da literatura. Ciênc. Saúde Col. 2016;20(9): 2731-47. http://dx.doi.org/10.1590/1413-81232015209.09562014

6. Carmo MML. Vivências de familiares cuidadores de pessoas com câncer: repercussões na dinâmica familiar e implicações para a Enfermagem. Viçosa. Trabalho de Conclusão de Curso [Graduação em Enfermagem] - Universidade Federal de Viçosa; 2017.

7. Minayo MCS. O desafio do conhecimento: pesquisa qualitativa em saúde. $14^{a}$ ed. São Paulo: Hucitec-Abrasco; 2014.

8. Pires AP. Amostragem e pesquisa qualitativa: ensaio teórico e metodológico. In: Poupart J. A pesquisa qualitativa: enfoques epistemológicos e metodológicos. Petrópolis: Editora Vozes; 2008.

9. Bardin L. Análise de conteúdo. 3a reimp da 1a ed. São Paulo: Edições; 2016.

10.Brasil. Conselho Nacional de Saúde. Resolução 466/12. Trata de pesquisas em seres humanos e atualiza a resolução 196. Diário Oficial da União. 12 dez. 2012. Disponible: conselho.saude. gov.br/resolucoes/2012/Reso466.pdf

11.Piolli KC, Medeiros M, Sales CA. Significations of being the caregiver of the companion with cancer: an existential look. Rev. bras. Enferm. 2016;69(1):99-105.

http://dx.doi.org/10.1590/0034-7167.2016690115i

12.Moreira NS, Souza CS, Poveda VB, Turrini RNT. Autoestima dos cuidadores de doentes oncológicos com capacidade funcional reduzida. Esc. Anna Nery. 2015;19(2):316-22. http://dx.doi.org/10.5935/1414-8145.20150043

13.Brasil. Política Nacional de Humanização: Ministério da Saúde; 2013. Disponible: http:// bvsms.saude.gov.br/bvs/publicacoes/politica_nacional_humanizacao_pnh_folheto.pdf

14.Machado BM, Dahdah DF, Kebbe LM. Cuidadores de familiares com doenças crônicas: estratégias de enfrentamento utilizadas no cotidiano. Cad. Bras. Ter. Ocup. 2018;26(2):299-13. https://doi.org/10.4322/2526-8910.ctoA01188

15.Araujo BN, Cantele A, Mingotti G. Acolhimento do enfermeiro aos familiares de portadores de câncer: a percepção do familiar. Rev. Saúde e Desen. 2017;11(9):144-55. Disponible: https://www.uninter.com/revistasaude/index.php/saudeDesenvolvimento/article/view/783 
16. Oliveira PSD, Viana FCA, Soares AKT, Andrade DLB. Assistência paliativa na ótica do cuidador familiar de paciente oncológico. Rev. Rede Cuid. Saúde. 2017;11(1):1-12. Disponible: http://publicacoes.unigranrio.edu.br/index.php/rcs/article/view/4262

17.Schimiguel J, Cenciarelli EA, Nunes LC, Lucena AF, Nosow V. O acolhimento de pacientes oncológicos: uma revisão bibliográfica. Saúde em Revista. 2015;5(39):47-7. http://dx.doi.org/10.15600/2238-1244/sr.v15n39p47-57

18.Ferreira PC, Wakiuchi J, Baldissera VDA, Sales CA. Sentimentos existenciais expressos por usuários da casa de apoio para pessoas com câncer. Esc. Anna Nery. 2015;19(1):66-2. http://dx.doi.org/10.5935/1414-8145.20150009

19. Wakiuchi J, Bennedeti GMS, Casado JM, Marcon SS, Sales CA. Sentimentos compartilhados por acompanhantes de pacientes oncológicos hospedados em casas de apoio: um estudo fenomenológico. Esc. Anna Nery. 2017;21(1):1-8. http://dx.doi.org/10.5935/1414-8145.20170011

20.Oliveira WT, Sales CA, Fernades CAM, Haddad MCL. Avaliação do bem-estar de cuidadores familiares de adultos com neoplasia maligna no âmbito domiciliar. Rev. Elet. Enferm, 2015;17(2):340-49. https://doi.org/10.5216/ree.v17i2.28714

21.Aoun SM, Deas K, Howting D, Lee G. Exploring the Support Needs of Family, Caregivers of Patients with Brain Cancer Using the CSNAT: A Comparative Study with Other Cancer Groups. Plos One. Jour. 2015;17(10):1-17. https://doi.org/10.1371/journal.pone.0145106

22.Dahdah DF, Carvalho AMP, Delsim JC, Gomes BR, Miguel VS. Grupo de familiares acompanhantes de pacientes hospitalizados: estratégia de intervenção da Terapia Ocupacional em um hospital geral. Cad. Terap. Ocup. 2013;21(2):399-04. https://doi.org/10.4322/cto.2013.041

23.Machado EP. Espiritualidade e saúde: uma dimensão de cuidado na vida de cuidadores familiares de pessoas com doença crônica. Viçosa. Tese [Doutorado em Ciências da Religião] - Pontifícia Universidade Católica de Goiás, Goiânia; 2014. Disponible: http://tede2.pucgoias.edu.br:8080/handle/tede/764

24.Silva BS, Costa EE, Gabriel IGSPS, Silva AE, Machado RM. Percepção de equipe de enfermagem sobre espiritualidade nos cuidados de final de vida. Cogit.Enferma. 2016;21(4):16. http://dx.doi.org/10.5380/ce.v21i4.47146

25.INCA (Instituto Nacional de Câncer). Cadernos de Psicologia: Os tempos no hospital oncológico. Brasil. Rio de Janeiro: INCA; 2015. Disponible: http://bvsms.saude.gov.br/bvs/ publicacoes/cadernos_psicologia_tempos_hospital_oncologico.pdf 